\title{
ENDOMETRIOSIS VESICAL. REVISIÓN DE LA LITERATURA, CON ESPECIAL REFERENCIA A LA ESPAÑOLA Y APORTACION DE DOS NUEVOS CASOS.
}

\author{
Hector Pastor Navarro, María José Donate Moreno, José Miguel Giménez Bachs, \\ José María Pastor Guzmán, Lorenzo Polo Ruiz, M. Atienzar Tobarra', Antonio Salinas Sánchez y \\ Julio Antonio Virseda Rodríguez.
}

Servicio de Urología. Servicio de Anatomía Patológica'. Hospital General Universitario. Albacete. España.

\begin{abstract}
Resumen.- OBJETIVO: Estudio y revisión de las hipótesis etiopatógenicas de la enfermedad endometriósica y de la clínica, diagnóstico y tratamiento de la endometriosis vesical.
\end{abstract}

MÉTODOS/RESULTADOS: Se revisa la literatura internacional, se recogen los casos publicados por autores españoles y se aportan dos casos propios.

CONCLUSIONES: La endometriosis vesical es una entidad patológica poco frecuente, pero hay que pensar en su diagnóstico en mujeres en periodo fértil con síntomas urinarios coincidentes con la menstruación. Su diagnóstico se efectúa con diversos métodos de imagen (ecografía, TAC, RMN) y se confirma con cistoscopia y biopsia. El tratamiento es quirúrgico (RTU, cistectomía parciall y médico (hormonal).

Palabras clave: endometriosis, vejiga, sindrome miccional, algia pélvica.
Summary.- OBJECTIVES: To study and review the etiopathogenic hypothesis for endometriosis and the clinical features, diagnosis and treatment of bladder endometriosis.

METHODS/RESULTS: We report two cases and review the international bibliography, collecting the cases published by Spanish authors.

CONCLUSIONS: Bladder endometriosis is a rare pathological entity, but its diagnosis should be considered in fertile women with urinary tract symptoms during menstruation. Diagnosis is obtained by various imaging tests (ultrasound, CT scan, MRI), and is confirmed with cystoscopy and biopsy. The treatment may be surgical (TUR, partial cystectomy) and/or medical (hormonall.

Keywords: Endometriosis. Bladder. Voiding symptoms. Pelvic pain.

\author{
Hector Pastor Navarro \\ Servicio de Urología \\ Hospital General Universitario \\ Hermanos Falcó, s/n \\ 02006 Albacete. (España) \\ Trabajo recibido: 20 de octubre 2005
}

\section{INTRODUCCIÓN}

Se denomina endometriosis a la existencia de tejido endometrial funcionalmente activo fuera de su localización habitual en la cavidad uterina. Se utiliza también el sinónimo de endometrioma para expresar un comportamiento clínico preferentemente tumoral o invasivo localizado en un determinado órgano. Es una enfermedad benigna, pero de comportamiento clínico a veces agresivo. Se da con mayor frecuencia en mujeres jóvenes, con media de edad entre 25-35 años. 


\section{MATERIAL Y MÉTODOS}

Se revisa la literatura internacional haciendo mención de las distintas teorías etiopatogénicas manejadas por distintos autores y de las diferentes denominaciones utilizadas para designar los tejidos en que se diferencia el sistema mulleriano secundario. Se recopilan todos los casos de endometriosis vesical publicados por autores españoles y se aportan dos nuevos casos de endometriosis vesical, exponiendo la clínica de presentación, el diagnostico y el tratamiento.

La endometriosis de la vejiga es una entidad infrecuente, aunque es el órgano del aparato urinario más veces afectado. Suele manifestarse clínicamente por síndrome miccional y algias pélvicas, de forma cíclica. Los métodos de imagen no son concluyentes para llegar al diagnostico definitivo. La cistoscopia es la prueba diagnóstica mas recomendable, aunque solo la histopatología dará el diagnostico de confirmación.

Presentamos dos casos, uno en mujer de 34 años, tratada con RTU y análogos de LH-RH, y la segunda de 31, tratada exclusivamente con RTU, permaneciendo ambas asintomáticas y sin evidencia de enfermedad, a los 5 y 9 años respectivamente.

\section{Caso Clínico 1}

Paciente con antecedentes de hernia de hiato y dos cesáreas a los 22 y 24 años. A los 31 años consultó por referir desde la segunda cesárea y cada vez con mayor intensidad, cuadro de síndrome mic-

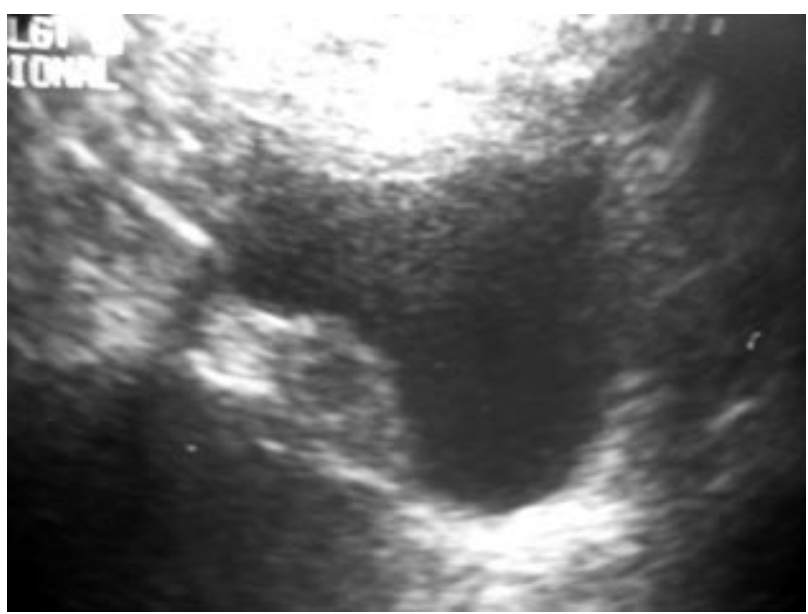

FIGURA 1. Ecografía abdominal con formación ecogénica intravesical. cional, acompañado de algias pelvianas y perineales, en los días previos, durante y posteriores a la menstruación. No refería hematurias ni fiebre. A la exploración los genitales externos y el abdomen no mostraban alteraciones. Fue tratada en múltiples ocasiones con antisépticos urinarios, aunque nunca se demostraron urocultívos positivos. La ecografía renal era normal y a nivel de vejiga se apreció en cara posterior una imagen excrecente, hiperecogénica de $1,5 \mathrm{~cm}$. Se realizó cistoscopia a mediados del ciclo menstrual describiéndose la existencia de pequeña área, mínimamente sobreelevada, deslustrada, retrotrigonal, que al contacto con el cistoscopio produce equímosis tenues, no identificandose en aquel momento como la imagen de la endometriosis.

La paciente se perdió para controles durante 3 años, durante los cuales continuó con la misma sintomatología, siendo tratada por su ginecólogo quien efectuó laparoscopia diagnostica no apreciando focos endometriósicos pélvicos, pero sí cuadro adherencial a paredes pélvicas y vejiga solidaria a cuerpo uterino.

La paciente acudió de nuevo a nuestro servicio, realizandose ecografía y TAC. En ecografía abdominal (Figura 1), y transvaginal se aprecia formación ecogénica en suelo vesical $2 \times 1,5 \times 2,7$.

En TAC abdominopélvico (Figura 2), no se observó patología a nivel de órganos abdominales, comprobándose en vejiga la presencia de una masa de $2 \times 3 \mathrm{~cm}$ en cara posterior sin plano de diferenciación claro con el útero.

A los 34 años y con la sospecha de posible endometriosis se realizó endoscopia vesical bajo

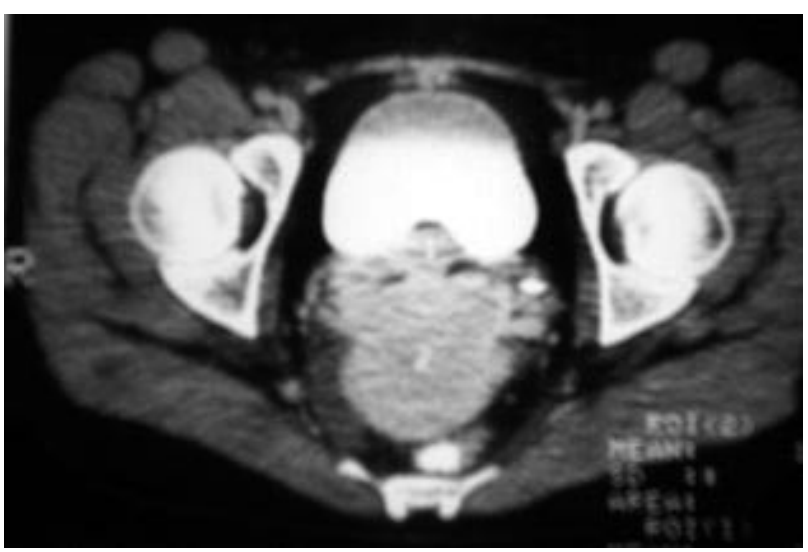

FIGURA 2. TAC abdominopélvico con masa de $2 \times 3$ $\mathrm{cm}$ en cara posterior vesical. 
anestesia, pocos días antes de la menstruación, observándose masa de aspecto morular de unos $3 \mathrm{~cm}$, retrotrigonal, con mucosa algo edematosa, íntegra y con áreas de color azulado. Se practicó RTU, mostrando al seccionar con el asa pequeñas cavidades, que se correspondían con glándulas endometriales quistificadas, de las que al corte manaba sangre retenida, en forma de fluido negruzco.

El estudio histopatológico (Figura 3), confirmó la presencia de nidos intramusculares de glándulas de tipo endometrial, rodeados de estroma endometrial edematoso, sin signos de malignidad; todo ello compatible con el diagnóstico de endometriosis.

La paciente fue tratada durante 6 meses con análogos de LH-RH. Cinco años después esta completamente asintomática y con varias ecografías vesicales y dos cistoscopias durante este periodo sin evidencia de recidiva.

\section{Caso Clínico 2}

Paciente de 31 años, con antecedentes de amigdalectomía, alergia a penicilinas, dos embarazos normales, con un parto eutócico a los 18 años, un aborto legrado a los 22 y una cesárea a los 24 . La paciente refería dismenorrea desde siempre. En una revisión ginecológica y con exploración normal, se efectuó ecografía donde se apreció aparato genital normal, y una imagen excrecente en cara posterior vesical de dos centímetros, sugestiva de tumor vesical (Figura 4).

La cistoscopia mostró neoformación polipoidéa, con áreas quísticas, con ligero tinte azulado, de dos $\mathrm{cm}$ de diámetro, localizado algo por encima y a la izquierda de orificio ureteral izquierdo. Se practicó RTU de la neoformación, describiéndose la histopatología como: Fragmentos de pared vesical revestidos por urotelio sin alteraciones relevantes. En la lámina propia y en la muscular subyacente se encuentran estructuras glandulares, dispersamente distribuidas, sin signos de atípia, que remedan las glándulas endometriales, rodeadas por estroma también de tipo endometrial. A veces el epitelio de dichas glándulas es de tipo mucoso endocervical, o bien es cuboidéo o cilíndrico ciliado. Focalmente hay acúmulo de macrófagos con pigmento parduzco intracitoplasmático. (Figuras 5 y 6 )

Posteriormente fue controlada por ginecología, aunque nunca se prescribió ningún tratamiento, efectuándose varias ecografías ginecológicas, vesicales y RMN pélvica sin hallar patología pélvica ni vesical. Nueve años después de la RTU, la paciente permanece asintomática, aunque sigue presentando dismenorrea, con dolor pélvico intenso los dos últimos días de la menstruación. En controles ginecológicos recientes con ecografía transvaginal y $R M N$ pélvica no se aprecia patología.

\section{DISCUSIÓN}

La endometriosis puede manifestarse en cualquier mujer desde la menarquia a la menopausia, con mayor incidencia en la segunda y tercera década de la vida, estimándose que existe endometriosis en el 3-10\% de las mujeres en edad fértil entre los 20 y 45 años, y hasta el 25-35\% en las infértiles (1 - 3), siendo el proceso ginecológico mas frecuente (2, 3). Su presentación en la menopausia (4) es muy in-

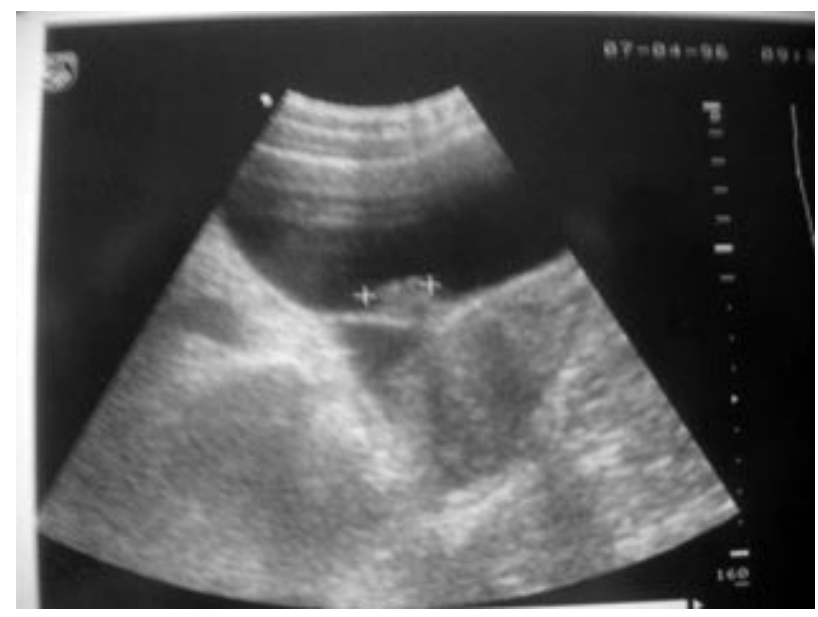

FIGURA 4. Ecografía abdominal con imagen intravesical de $2 \mathrm{~cm}$
FIGURA 3. Glándulas y estroma endometrial en el espesor de la muscular. HE.

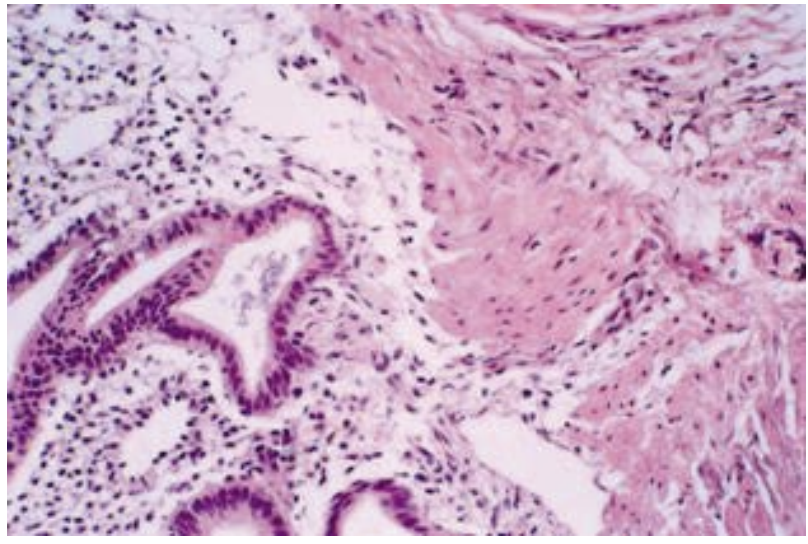


frecuente, pudiendo estar en relación con el aumento de estrógenos circulantes, por aportación externa o por aumento de la producción endógena $(5,6)$, ya sea de origen ovárico o suprarrenal, debiendo descartarse tumores de estos órganos. Otra causa relacionada con niveles séricos elevados de estrógenos sería por aumento de la conversión periférica, mediada por aromatasas, de androsténdiona a estrona, especialmente en casos de obesidad. La afectación urológica ocurre en el 16-24 \% de las mujeres endometriósicas, pero se estima que solo manifiestan sintomatología urológica entre el $1-2 \%(2,7,8)$. El número de casos publicados, sin embargo, es mucho menor de lo que correspondería a estas cifras.

La endometriosis ha sido descrita en muy diversas localizaciones, algunas muy alejadas de la cavidad pélvica como pulmón, piel y aparato digestivo. La localización pélvica es la mas frecuente, afectando a ovario $55 \%$, ligamento ancho (35\%), fondo de saco $(35 \%)$ y ligamentos uterosacros $(8 \%)(9,10)$. Dentro de la localización en el aparato urinario la vejiga se afecta en el $84 \%$, el uréter en el $7-15 \%$, el riñón $4 \%$ y la uretra en el $2 \%(2,3,11)$.

El endometrio ectópico tiene la capacidad de crecer, infiltrar e incluso diseminarse, en una forma similar a como lo hace un tejido neoplásico $(6,10)$.

Hay autores que diferencian entre endocervicosis, endometriosis y endosalpingiosis, en relación al hallazgo histológico de endometrio propio del cervix, útero o trompas en situación ectópica $(2,12$, 13). Todas serían entidades clínicas relacionadas por su origen embriológico común, tratándose de una di- ferenciación no maligna del sistema mulleriano secundario (mullerianosis), procedente de la cavidad celómica del embrión, y que se diferencia posteriormente en endometrio, endocervix o tejido tubárico (12-16). La denominación de endometriosis, endocervicosis o endosalpingiosis se aplica por existir uno solo de estos tejidos o por un predominio de uno de ellos sobre los otros; a nivel vesical es frecuente encontrarlas asociadas y en estos casos se puede encontrar habitualmente antecedentes de endometriosis pélvica o cirugías previas por esta causa. En nuestro segundo caso están presentes los tres tejidos en que se puede diferenciar el sistema mulleriano secundario, el endometrial, el endocervical y el endosalpingial, aunque predominio del primero, se trata pues de una endometriosis pero con componente de endocervicosis y endosalpingiosis. De los 6 casos de endocervicosis que presenta Clement PB y Young RH (13) en 1992, 3 tenían asociados focos de endometriosis y uno de endosalpingiosis en el estudio histológico, y otro coexistía con endometriosis pélvica; la forma aislada mas rara es la endosalpingiosis vesical, al parecer por que produce menos síntomas.

Para explicar la existencia de endometrio ectópico o enfermedad endometriósica se manejan tres teorías etiopatogénicas:

- Embrionaria: Propuesta por Von Recklinghausen (17) (1896), que postula que los tejidos endometriales ectópicos se desarrollarían a partir de restos embrionarios de los conductos de Wolf, y por Cullen $(6,18)(1896)$ y Kossman (19) $(1897)$ de los de Muller $(7,12,20-24)$, que explicaría los casos de presentación en varones tratados con estrógenos $(7,23)$.

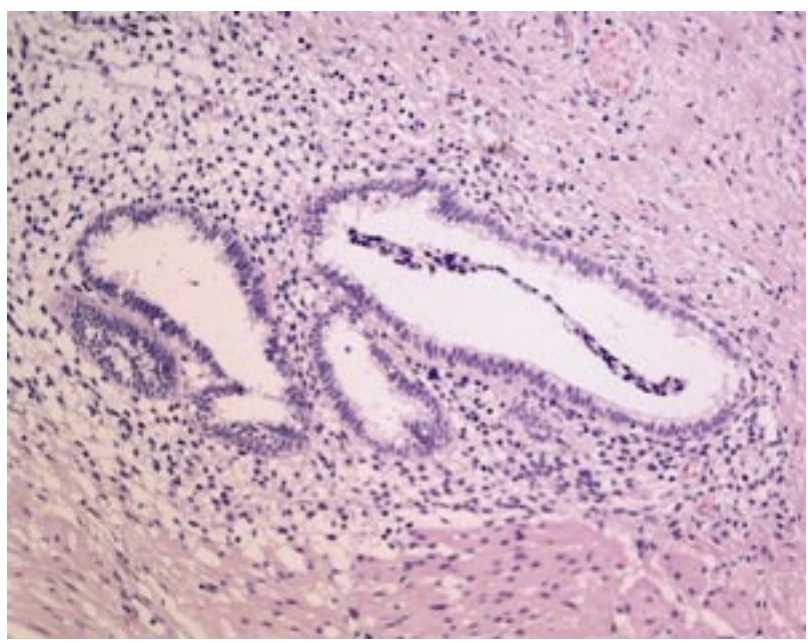

FIGURA 5. Glándulas y estroma de tipo endometrial en el seno de la muscular propia vesical. HE.

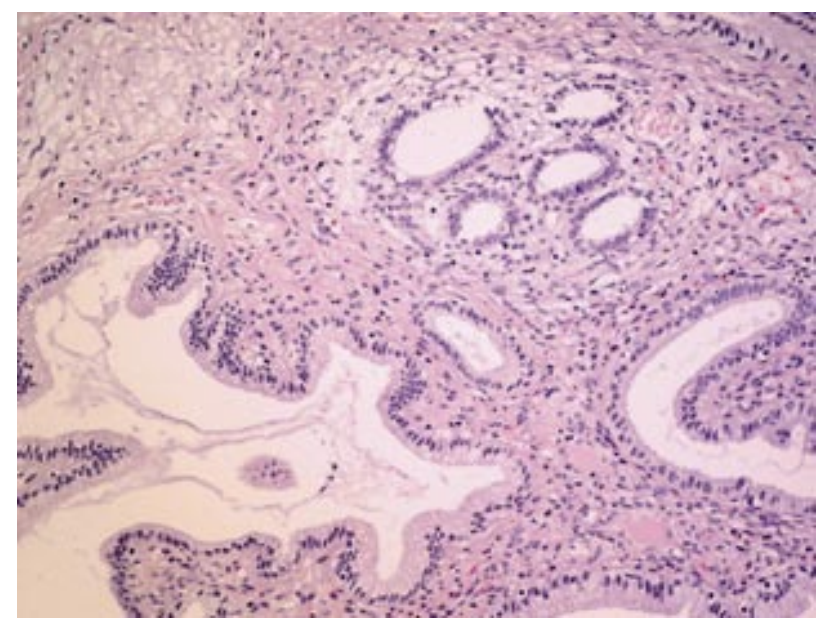

FIGURA 6. Glándulas revestidas por epitelios endometrial y endocervical en el seno de la pared vesical. HE. 
- Metaplásica: Propuesta en 1898 por Iwanoff (25), elaborada por Meyer (26) (1924) y sugerida por Dreyfus (27) y Kretschmer (28), ya que el epitelio endometrial y la serosa peritoneal proceden embriológicamente del mesotelio celómico y por ello cualquier célula del mismo origen, estimulada por factores desconocidos probablemente hormonales o inflamatorios, podrían transformarse en células endometriales, incluso a nivel del epitelio vesical $(12,22,24,29)$.

- Migratoria o metastásica: Propuesta por Cullen $(7,18,28)$ en 1897; Sampson avanza la teoría de la implantación con la menstruación retrógrada (30), y Jacobson (31) en 1922 induce experimentalmente endometriosis en animales tras la implantación de tejido endometrial en cavidad peritoneal y vejiga $(7,22,28,30,31)$. La menstruación retrógrada de tejido endometrial $(30,32)$, a través de las trompas podría alcanzar cualquier lugar del aparato genital femenino e incluso pared vesical, implantándose en estos lugares. Esta migración celular, es posible por embolización linfática o hematógena ya que se ha hallado tejido endometrial en venas y linfáticos $(6,7,12,22,33,34)$, y explicaría la existencia de los focos distantes de la pelvis; también puede haber invasión por contigüidad o extensión directa a través del intersticio, desde la pared uterina $(7,18,28,34)$. Esta difusión del tejido endometrial a otros territorios puede producirse en el curso de histerosalpingografías o de cirugía pélvica del aparato reproductor $(2,9,11,12,34-36)$, particularmente en el caso de cesáreas, donde la localización vesical suele ser única. Así mismo se ha especulado que en algún caso podría haber ascenso de tejidos de menstruación por vía uretral e implantación vesical como ocurre en las infecciones urinarias.

Todas estas teorías, pero especialmente la migratoria por implantación directa o metastásica hacen suponer que gran parte de las endometriosis vesicales podrían originarse a partir de focos endometriósicos preexistentes en la pelvis $(11,29)$. Henriksen (37) distingue la endometriosis vesical primaria de la secundaria; solo se aceptaría la primera si se demuestra que no es una prolongación de otro foco endometriósico vecino, y no hay antecedente de cirugía pélvica $(24,34,37)$. En el $12 \%$ de las pacientes la lesión o lesiones vesicales, pueden ser la única localización de la enfermedad (6). Actualmente, se tiende a resumir y considerar que existen dos formas etiopatogénicas de endometriosis vesical:

- Espontánea, en el curso de una enfermedad pélvica difusa.

- Vesical única o aislada, por siembra iatrógena tras intervenciones ginecológicas, especialmente postcesárea $(5,10,34,35,38,39)$.
En 1860, Rokitansky define por primera vez la endometriosis $(2,40)$, tras observaciones de autopsias. Desde que en 1921 E.Starr Judd (41) describió el primer caso de endometriosis con afectación vesical, han sido publicados hasta el momento alrededor de 350 casos de endometriosis vesical, de los que 30 son españoles (Tabla 1), a los que hay que sumar los dos que aportamos en el presente trabajo. La endocervicosis pélvica fue descrita por primera vez por Goodall y cols. (42) en 1943; su localización vesical se publicó por Clement y Young (13) en 1992, existiendo actualmente tres decenas de casos publicados, 14 de localización vesical $(13,15,16)$, dos de ellos en la literatura nacional $(12,14)$; nuestro segundo caso podría ser el tercer caso de endocervicosis en nuestro país, pues a pesar del predominio de glándulas endometriales, también existen de endocervix. La endosalpingiosis la describió Sampson (43) en 1928, estando documentada en diversas localizaciones en unos cien casos, pero solo con afectación vesical en 8 casos, uno publicado por autores españoles (44). El segundo caso que presentamos también tiene componente de epitelio tubárico.

La sintomatología provocada por un endometrióma vesical, puede ser muy variada y dependerá de la localización del mismo, de su tamaño y del momento del ciclo menstrual. El asiento más frecuente es en trígono y cúpula. Las lesiones pequeñas pueden cursar de forma asintomática y diagnosticarse tras exploración ginecológica por otras patologías. La enfermedad intrínseca, es decir la que afecta al detrusor dará sintomatología en el $75 \%$ de los casos, casi siempre cíclica $(6,12,24)$ y más exacerbada en los días perimenstruales. Básicamente se manifiesta con un síndrome miccional con polaquiuria, tenesmo, escozor, dolor miccional, disuria, molestias y dolor suprapúbico. La endometriosis pélvica es la responsable en el 25-35\% de las algias pélvicas crónicas cíclicas secundarias (45), siendo mas intenso el dolor cuanto mas profunda sea la penetración de las lesiones. La hematuria coincidente con la menstruación (menuria), no es tan constante como el síndrome miccional, apareciendo solo en el 20-25 \% de casos, cuando existe afectación de la mucosa $(5,7,20,29,34,46)$. Tanto la clínica miccional como la dolorosa parecen producidos por la acumulación progresiva de restos menstruales no expulsados y con la extensión de las lesiones a la capa muscular $(11,12,34)$. La demora media en el diagnostico correcto es de 4-5 años, a pesar de la evidencia en una mayoría de casos, de la sintomatología cíclica $(7,12)$.

En el $50 \%$ de casos existe antecedente de cirugía pélvica, especialmente de cesáreas $(5,9,10,34$ $36,38-40)$, pues tras la disrupción del útero hay siembra endometrial y posterior infiltración de pericisto, detrusor y mucosa, por este orden. 
Los trastornos del sistema inmunitario podrían desempeñar algún papel en la etiopatogenia de la endometriosis (3). La dependencia hormonal del tejido endometrial se puede confirmar histológicamente con técnicas inmunohistoquímicas utilizando anticuerpos antireceptores de estrógenos y progesterona (47). Se ha descrito un aumento de CA-125 en suero de mujeres endometriosicas, que es un antígeno presente aunque no específico en todo epitelio derivado del conducto de Muller, tanto en el normal como en las anomalías que de el se deriven, ya sean benignas o malignas $(3,8,12,13,38,48)$, habiendo sido utilizado sobre todo para control de la eficacia del tratamiento.

Para el diagnostico tanto la ecografía abdominal o vaginal, TAC, y RMN pondrán de manifiesto la masa, pero no son especificas; la TAC pero especialmente la $R M N$, define y delimita más exactamente la afectación y profundidad de la lesión en la pared vesical y sirve como estudio de extensión, para buscar mas focos endometriósicos sobre todo a nivel pelviano; también puede ser necesaria la laparoscopia para descubrir focos de endometriosis abdominal coexistentes. La exploración más rentable es la cistoscopia. La primera descripción cistoscópica de la endometriosis la hizo Muller (49) en 1927 observando una lesión angiomatosa vesical, descubriéndose en su estudio histopatalógico que se trataba de una endometriosis (34), pero fue Ottaw (50) en 1929 el que hizo el primer diagnostico endoscópico preoperatorio. La imagen cistoscópica de la endometriosis se modificará a lo largo del ciclo menstrual, siendo más nítida y característica durante la menstruación, al aumentar de tamaño y hacerse más congestiva la lesión, pudiendo observarse una tumoración sólida con tapizado hiperhémico, con bullas o quistes azulados o violáceos en su superficie, levemente sobreelevados, rodeados de un halo congestivo y edematoso. Cuando las lesiones son pequeñas a veces es preciso efectuar mas de una cistoscopia en distintos momentos del ciclo $(23,24,34)$. Si solo afecta a la adventicia vesical, la cistoscopia no será diagnóstica. El diagnostico de confirmación es histológico tras biopsia fría o RTU de la masa $(21,22)$.

Como la sintomatología urinaria puede ser similar a la de cistitis de repetición, cistitis intersticial, carcinoma "in situ", o tuberculosis, habrá que hacer diagnostico diferencial con todos estos procesos $(1,3,34)$. Cuando se haya demostrado la lesión - la masa vesical por los métodos de imagen y/o cistoscopia el diagnostico diferencial se hará con el carcinoma vesical, angiomas, leiomioma, cistopatías (amiloidosis, malacoplaquia, cistitis glandular, adenoma nefrógeno),y procesos extravesicales como la diverticulitis $(10,12,23)$, siendo necesario recurrir al estudio histológico, en casi todas las ocasiones.
La malignización de la endometriosis es muy poco frecuente, solo está bien documentada en el 0,3-0,8 \% de casos (29,51-55) de endometriosis ovárica. Se podrá considerar así, cuando existan tejidos endometriales malignos en el seno de estructuras endometriales típicas y normales, y se compruebe que no es una metástasis, de otro foco primario. Hay siete casos descritos (52-55), seis adenocarcinomas y un adenosarcoma vesicales, de probable origen endometriósico, que en caso de ser así, el porcentaje de malignización se aproximaría al $2 \%$.

El tratamiento deberá ser individualizado, dependiendo de la edad, del deseo de futuros embarazos, la intensidad de la clínica, de la localización o de si hay más órganos involucrados. Dos tipos de tratamiento son posibles: medico-hormonal y quirúrgico. Con el tratamiento hormonal se obtiene una muy buena respuesta inicial, con grado alto de tolerancia por las pacientes, y con posibilidad de suspender el tratamiento, si hubiera efectos secundarios que lo requirieran. Se ha aplicado tratamiento hormonal con estrógenos y progesterona asociados (5) en lesiones vesicales pequeñas con remisión total o parcial de los síntomas en porcentajes elevados. Está bien establecido, de todas formas, que con tratamiento médico exclusivo, la endometriosis profunda y severa no se cura definitivamente, pues solo induce desaparición temporal de las lesiones activas (32) , llegando las tasas de recidivas en endometriosis pélvicas al $56 \%(12,56,57)$. El mas habitual y con mejor pronóstico, teniendo en cuenta su hormonodependencia, es quirúrgico (cistectomía parcial, RTU) y médico a la vez (hormonal).

Algunos autores consideran que la RTU esta contraindicada $(4,34,56)$, pues la lesión en muchos casos interesa la pared vesical y pericisto, por lo que este método sería insuficiente e incluso peligroso por la posibilidad de perforación vesical; sin embargo la RTU-biopsia del endometrioma, que nos aportará el diagnostico de confirmación, y el bloqueo hormonal con análogos de la LH-RH, es el tratamiento de inicio mas veces utilizado en los últimos años, aunque hay que tener en cuenta que el porcentaje de recidivas se estima entre el 25-35\% (58). Desde la aparición de los análogos, estos son el tratamiento médico de elección, habiendo caído en desuso los estrógenos, andrógenos, progestágenos y danazol, empleados antaño, por provocar mas efectos secundarios. Los análogos inducen un estado anovulatorio similar al postmenopaúsico, un hipogonadismo hipogonadotropo, descendiendo la concentración sérica de estrógenos a niveles de castración, que hace regresar el tejido endometrial $(3,12)$.

Otros tratamientos quirúrgicos pueden ser a veces necesarios, como es la cistectomía parcial 
$(1,7,11)$, tanto por laparotomía como por laparoscopia (59) para la exéresis completa, ya que la lesión en muchos casos es transmural, como ocurre tras la cirugía pélvica ginecológica, especialmente tras cesáreas. La castración quirúrgica, ooforectomía bilateral e histerectomía, es el tratamiento definitivo, indicado cuando no se desea conservar la fertilidad o en pacientes de edad avanzada. Otra opción muy poco utilizada es la radioterapia de los ovarios y de los focos endometriósicos, con el fin de tratar la enfermedad y suprimir la actividad hormonal; su indicación quedaría limitada a enfermas de elevado riesgo quirúrgico $(12,29)$.

Si el diagnostico de endometriosis vesical se produce en mujeres próximas a la menopausia, no se deberán aplicar tratamientos agresivos, si no esperar a que ésta se presente, pues las lesiones regresarán espontáneamente la mayor parte de las veces $(10,12,29,60)$.

Según diversas publicaciones recientes, la modalidad terapéutica mas usada es la cirugía, mediante la RTU mas hormonoterapia con LH-RH, con buenos resultados y desaparición de la clínica en la mayoría de las enfermas $(2,39,57,58,61)$, como ocurrió en nuestro primer caso. Así mismo parece no existir recidiva clínica en más del $40 \%$ de casos de endometriosis pélvicas tratadas solo con hormonoterapia. Pero no esta claramente explicado por qué ocurre esta evolución tan favorable, sobre todo en pacientes en edad fértil, si en muchas ocasiones la lesión vesical es transmural y el bloqueo hormonal es temporal. Posiblemente pueda tener algún lugar en el tratamiento médico conservador los progestágenos de aplicación local como el DIU con levonorgestrel, pues aunque es utilizado en ginecología como anticonceptivo y para la menorragia idiopática, es eficaz en focos endometriósicos pélvicos, como en septo vesicovaginal $(32,62)$, posiblemente por alcanzar altas concentraciones del fármaco a nivel del endometrio y en áreas próximas, aunque con bajas concentraciones plasmáticas, con dos importantes ventajas: es eficaz durante cinco años y no altera la fertilidad futura tras la interrupción del tratamiento.

Hemos encontrado 30 casos publicados por autores españoles, que junto a los dos del presente trabajo hacen un total de 32 (Tabla I).

El primero de ellos se describe en la primera edición del libro Cistitis y cistopatías de 1947, de L. Cifuentes Delatte (24), siendo un diagnostico clínico y endoscópico, pues la biopsia que se efectuó con pinza fría no se pudo procesar por extravío de la misma (su primera biopsia endoscópica según relata el autor); por las fechas y años de seguimiento se deduce que el mismo caso es citado en la segunda edición del referido libro (1989), y en las dos ediciones de Cirugía Urologica Endoscópica laños 1961 y 1981) del mismo autor, pero ya tras RTU y estudio histológico.

El segundo caso fue publicado por Picatoste en 1957 (63), y era el caso número 77 de la literatura mundial en aquellas fechas.

Posteriormente pasan más de tres décadas para que se publiquen nuevos casos. En $1986 \mathrm{~J}$. A. Lopez Lopez (52) y cols. publican un caso de adenocarcinoma escamoso de vejiga con componente endometriode.

\section{A partir de 1990 se publica el resto de ca-}

sos.

La mayoría de los autores, -diecinueve-, aportan un solo caso. Dos casos presentan Silmi (29), Vicente $(23,64)$, Alapont (8), Martin A (59) y nosotros; tres Parras (65). En total son pues 32 casos de endometriosis vesical publicados por autores españoles.

El antecedente de cirugía ginecológica o cesárea se constata en muchos casos; varias ovariectomías previas por endometriosis, y cesáreas en 15 pacientes, en tres de ellas en dos ocasiones, por lo que el antecedente de cesárea se aproxima al 50\%. La media de edad de las pacientes españolas, si excluimos el caso de adenocarcinoma con diferenciación endometriode de J.A. López lópez (52), y otro caso en el que no consta, se sitúa en los 35 años.

En cuanto al tratamiento realizado predomina la RTU como tratamiento quirúrgico, (en 25 casos, 3 iterativas por recidiva) seguido de la cistectomía parcial ( 10 casos, en 5 tras recidiva post-RTU), efectuándose dos de ellas por laparoscopia (59). El tratamiento hormonal mas prodigado y casi siempre asociado a la cirugía fueron los análogos de LH-RH.

Como entidades patológicas próximas, también se han publicado por autores españoles dos casos de endocervicosis $(12,14)$, el primero con focos endometriósicos y el segundo con endometriosis ovárica, y uno de endosalpingiosis (44), aunque con pequeños focos endometriósicos. Existe otro trabajo firmado por autores españoles y franceses (48), en el que se describe la clínica y la inmunohistoquímica, y en el que aparece tejido tubárico, endometrial y de endocervix.

En comunicación en el LXX congreso Nacional de Urología, Romero Doncel y cols. (66), exponen la casuística de su servicio (no incluida en Tabla 1), 


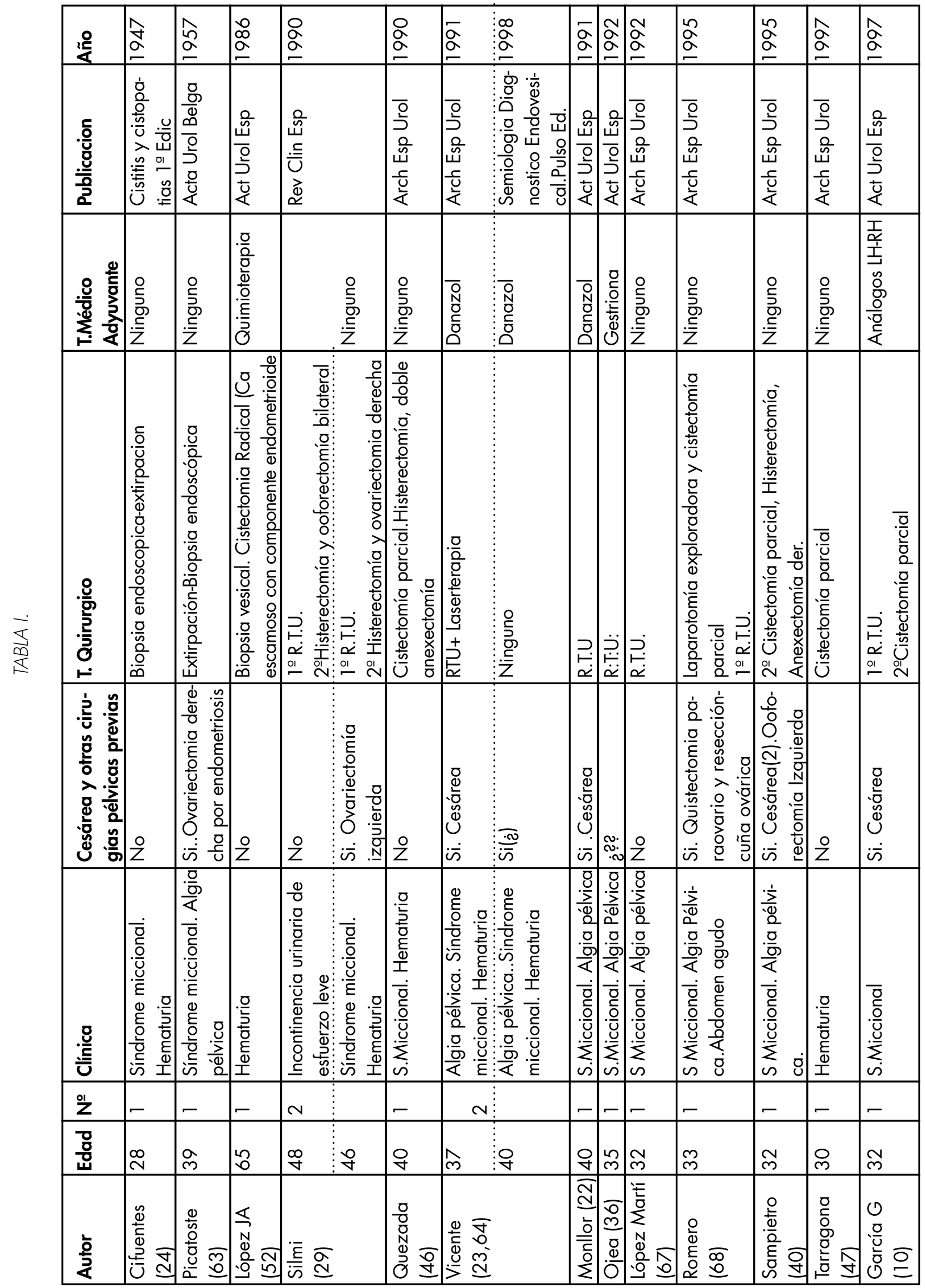




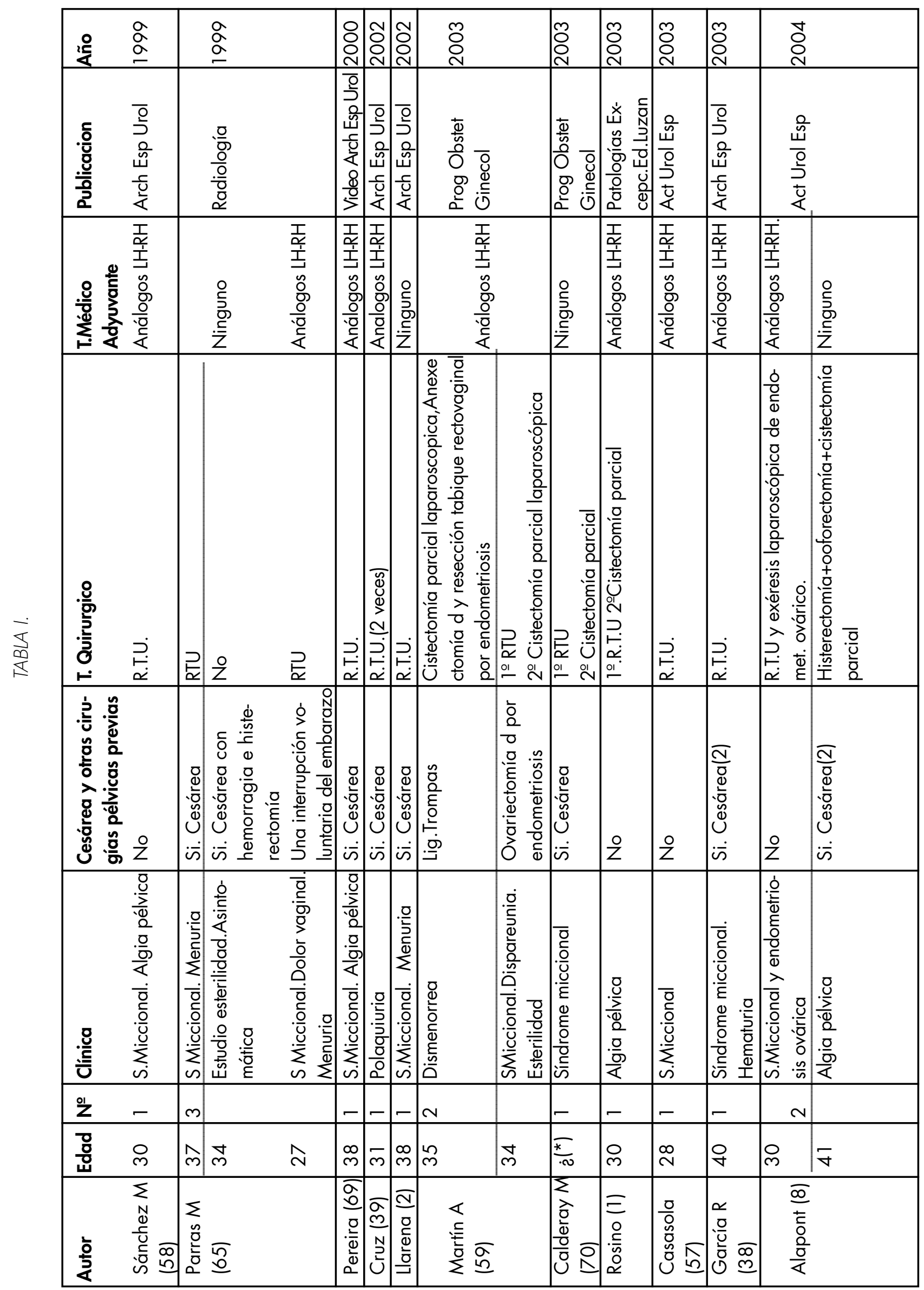


aportando cuatro casos mas de endometriosis vesical, con edad media de 33 años, en tres de ellos con antecedente de cesárea, tratándose en los cuatro casos con RTU, aunque se efectuaron dos cistectomías parciales en dos casos de recidiva; en dos pacientes se administró tratamiento adyuvante con análogos de LH-RH.

\section{BIBLIOGRAFÍA y LECTURAS RECOMENDADAS ( ${ }^{*}$ lectura de interés $y^{* *}$ lectura fundamental)}

1. ROSINO SANCHEZ, A.; GARCIA PEÑALVER, C.; DIAZ GONZALEZ, R.: "Endometriosis vesical. Patologías excepcionales en urología". Ed. Luzán, 55, 2003.

*2. LLARENA IBARGUREN, R.; LECUMBERRI CASTAÑOS, D.; PADILLA NIEVA, J. y cols.: "Endometriosis urinaria". Arch. Esp. Urol., 55: 1209, 2002.

**3. BOLOGNA, R.A.; WHITMORE, K.E.: "La endometriosis genitourinaria". Ball, T.P. editor. AUA update series. Edición Española, 1: 21, 2001.

4. VORSTMAN, B.; LYNNE, C.; POLITANO, V.A.: "Postmenopausal vesical endometriosis". Urology, 22: 540, 1983.

*5. WESTNEY, O.L.; AMUNDSEN, C.L.; McGUIRE, E.J.: "Bladder endometriosis: conservative management". J. Urol., 163: 1814, 2000.

**6. COMITER, C.V.: "Endometriosis de la vía urinaria. Urologia femenina". Clin. Urol. North Am.(Edic Española), 3: 635, 2002.

*7. SHOOK, T.E.; NYBERG, L.M.: "Endometriosis of the urinary tract". Urology, 31: 1, 1988. ALAPONT ALACREU, J.M.; ANDREU GAR-

8. CIA, A.; HERRERO POLO, E. y cols.: "Endometriosis vesical: dos nuevos casos". Act. Urol. Esp., 28: 789, 2004

9. ONG,C.L.; TUNG, K.H.: "Bladder endometriosis: three case reports and a review". Aust. NZ. J. Surg., 61: 81, 1991.

10. GARCIA GONZALEZ, J.I.; EXTRAMIANA CAMENO, J.; ESTEBAN CALVO, J.M. y cols.: "Endometriosis vesical tras cesárea: aspectos diagnósticos-terapéuticos". Act. Urol. Esp., 21: 785, 1997.

*11. STANLEY, K.E.; UTZ, D.C.; DOCKERTY, M.B.: "Clinical significant endometriosis of the urinary tract". Surg. Ginecol. Obstet., 120: 491, 1965.

*12. LEIVA, O.; ORTIZ VICO, F.: "Endocervicosis de la vía urinaria: sistema mülleriano secundario. $\mathrm{Pa}$ tologías excepcionales en Urología”. Ed. Luzán, 11, 1998.

*13. CLEMENT, P.B.; YOUNG, R.H.: "Endocervicosis of the urinary bladder. A report of 6 cases of benign mullerian lesion that may mimic adeno- carcinoma". Am. J. Surg. Pathol., 16: 533, 1992.

14. GONZALEZ SATUÉ, C.; SERRA CÁNER, J.; ARMORA MANÍ, J.: "Endocervicosis vesical: excepcional causa de masa vesical". Act. Urol. Esp., 28: 677, 2004.

15. SPENCER, S.; RUBIN, M.; HUSSAIN, H. y cols.: "Complete transuretral resection of bladder endocervicosis". J. Urol., 164: 254, 2001.

16. RODRIGUEZ, R.; ALFERT, H.: "Endocervicosis of the bladder: a rare mucinous analogue of endometriosis". J. Urol., 157: 1355, 1997.

17. VON RECKLINGHAUSEN, F.: "Die Adenomyome und Cist Adenomyiome der Uterus und Tuben Wandung, ihre abkunft von resten des Wolffschen Koerpers”. Berlin A. Airschwald, 247, 1896.

18. CULLEN, T.J.: "Adenomyoma of the rectovaginal septum”. Bull Johns Hopkins Hosp., 28: 343, 1917.

19. KOSSMAN.: "Die abstammung des druseneinschlusse in den adenomyomen des uterus und der tuben". Arch. f. Gynak, 54: 359, 1897.

20. FEIN, R.L.; HORTON, B.F.: "Vesical endometriosis a case report and review literature". J. Urol., 95: 45, 1966.

21. PRETICONI, R.; HAUTMANN, R.: "Problems diagnostics et therapeutiques des endometriosis de la vessie". J. Urol., 205, 1987.

22. MONLLOR GISBERT, J.; MERINO HERNAEZ, C.; OLIVER GOMEZ, C. y cols.: "Endometriosis vesical, aproximación diagnóstica y terapeútica". Act. Urol. Esp., 15: 86, 1991.

23. VICENTE, J.; LAGUNA, P.; DIAZ, I. y cols.: "Treatment of bladder endometriosis vising the Nd-Yag laser". Arch. Esp. Urol., 44: 169, 1991.

*24. CIFUENTES DELATTE, L.: "Endometriosis vesical.Cistitis y Cistopatias". $1^{\text {a }}$ Ed. Paz Montalvo, Madrid, 375, 1947.

25. IWANOFF, N.: "Drusiges cystenhaltiges uterus fibroyom compliziert durch sarkom und karzinom”. Monatschr. f. Geburtsch. u. Gynak, 7: 295, 1898.

26. MEYER, R.: "Die Bedeutung der heterotopen Epithelwuchnerung im Ovarium und am Peritoneum". Zentralb. f. Gynak, 14: 722, 1924.

27. DREIFUS, M.L.: "Internal endometriosis of the urinary bladder". Am. J. Obst. Gyn., 39: 336, 1940.

28. KRESTCHMER, H.I.: "Endometriosis of the bladder". J. Urol., 53: 459, 1945.

**29. SILMI MOYANO, A.; BLÁZQUEZ IZQUIERDO, J.; SALINAS CASADO, J. y cols.: "Endometriosis vesical. Revisión y presentación de dos casos". Rev. Clin. Esp., 186: 74, 1990.

30. SAMPSON, J.A.: "Peritoneal endometriosis due to menstrual disemination of endometrial tissue into the peritoneal cavity". Am. J. Obstet. Gynecol., 14: 422, 1927. 
31. JACOBSON, V.C.: "Autotransplantation of endometrial tissue in the rabbit". Arch. Surg., 5: 281, 1922.

**32. VERCELlinI, P.; FRONTINO, G.; PIETROPAOLO, G. y cols.: "Deep endometriosis: definition, pathogenesis, and clinical management". J. Am. Assoc. Gynecol. Laparoscop., 11: 153, 2004.

33. JAVERT, C.I.: "Pathogénesis of endometriosis based on endometrial horneoplasia, direct extensión, exfoliation and implantation, lymphatic and hematogenus metastasis (incluiding 5 cases reports of endometrial tissue in pelvic lymph nodes)". Cancer, 2: 399, 1949.

**34. ABESHOUSE, B.S.; ABESHOUSE, G.: "Endometriosis of the urinary tract". J. In. Coll. Surg., 43, 1960.

35. BUKA, N.J.: "Vesical endometriosis after cesarean section". Am. J. Obstet. Gynecol., 158: 1117, 1988.

36. OJEA CALVO, A.; RODRIGUEZ IGLESIAS, B.; ALONSO RODRIGO, A. y cols.: "Endometriosis vesical". Act. Urol. Esp., 6: 805, 1992.

37. HENRIKSEN, E.: "Primary endometriosis of the urinary bladder". J.A.M.A., 104: 1401, 1935.

38. GARCIA RODRIGUEZ, J.; FERNANDEZ GOMEZ, J.M.; JALON MONZON, A. y cols.: "A new case of bladder endometriosis in a patient with a history of cesarean section". Arch. Esp. Urol., 56: 952, 2003.

39. CRUZ GUERRA, N.A.; LINARES QUEVEDO, A.; CUESTA ROCA, C. y cols.: "Bladder endometriosis in a patient with cesarean history". Arch. Esp. Urol., 55: 194, 2002.

40. SAMPIETRO CRESPO, A.; FERNANDEZ DURAN, A.M.; RUIZ MONDEJAR, R. y cols.: "Bladder endometriosis: a new case and review of the literature". Arch. Esp. Urol., 48: 314, 1995.

41. JUDD, E.S.: "Adenomyoma presenting as a tumor of the bladder". Surg. Clin. North. Am., 1: $1271,1921$.

42. GOODDALL, J.R.: "A Study of Endometriosis, Endosalpyngosis, Endocervicosis and PeritoneoOvarian sclerosis". Philadelfhia: J.B. Lippincott Co., 89, 1943.

43. SAMPSON, J.A.: "Endometriosis following salpingectomy". Am. J. Obstet. Gynecol., 16: 461, 1928.

44. JIMENEZ-HEFFERMAN, J.A.; SANCHEZ-PIEDRA, D.; BERNALDO DE QUIRÓS, L. y cols.: "Endosalpingiosis( mullerianosis) of the bladder: a potential source of error in urinary cytology". Cytopathology, 11: 348, 2000.

45. CHAPRON, C.: "Doleur et endometriose profonde". J. Gynecol. Obstet. Biol. Reprod., 32: 32, 2003.

46. QUEZADA, F.; BERROCAL, A.; ESTEBAN, E. y cols.: "Endometriosis vesical". Arch. Esp. Urol., 43: 19, 1990.
47. TARRAGONA FORADA, J.; DE TORRES RAMIREZ, I.; MOROTE ROBLES, J. y cols.: "Vesical endometriosis. Report of a case with inmunohistochemical study". Arch. Esp. Urol., 50: 74, 1997.

48. DONNE, C.; VIDAL, M.; BUTTIN, X. y cols.: "Mullerianosis of the urinary bladder: clinical and inmunohistochemical findings". Histopathology, 33: 290, 1998.

49. MULLER, J.: "Endometrioide Adenomatose (Adenomyose) und Cystadenomatose der Harnblase". Arch. f. klin. Chir., 145: 394, 1927.

50. OTTAW, B.: "Dasklinische blid der endometriose der harnblase". Arch. Gynak., 3: 137, 1929.

51. MOSTOFIZADEH, M.; SCULLY, R.E.: "Tumores malignos originados en endometriosis". Clin. Obst. Ginec., 3: 973, 1980.

52. LOPEZ, J.A.; PERES-ARBÉS, J.A.; ESCIVÁ PLÁ, J. y cols.: "Adenocarcinoma escamoso de vejiga con componente endometriode, comentarios sobre un caso". Act. Urol. Esp., 10: 217, 1986.

53. VARA, A.R.; RUZICS, E.P.; MOUSSABECK, O. y cols.: "Endometrioid adenosarcoma of the bladder arising from endometriosis". J. Urol., 143: 813, 1990.

54. BALAT, O.; KUDELKA, A.P.; EDWARS, C.L. y cols.: "Malignant transformation in endometriosis of the urinary bladder: case report of clear cell adenocarcinoma”. Eur. J. Gynaecol. Oncol., 17: 13, 1996.

55. GARAVAN, F.; GRAINGER, R.; JEFFERS, M.: "Endometrioid carcinoma of the urinary bladder complicating vesical Mullerianosis: a case report and review of the literature". Virchows Arch., 44: 587, 2004.

56. PRICE, D.T.; MALONEY, K.E.; IBRAHIM, G.K. y cols.: "Vesical endometriosis: report of two cases and review of the literature". Urology, 48: 639, 1996.

57. CASASOLA CHAMORRO, J.; GUTIERREZ GARCIA, S.; FERNANDEZ ROJO, F. y cols.: "Endometriosis vesical. Diagnostico y tratamiento". Act. Urol. Esp., 27: 394, 2003.

**58. SANCHEZ MERINO, J.M.; GUILLAN MAQUIEIRA, C.; GARCIA ALONSO, J.: "Tratamiento de la endometriosis vesical. Revisión de la literatura española". Arch. Esp. Urol., 58: 189, 2005.

*59. MARTIN, A.; PLASENCIA, W.; GARCIA, R. y cols.: "Tratamiento laparoscópico de la endometriosis vesical”. Prog. Obstet. Ginecol., 46: 403, 2003.

60. HABUCHI, T.; OKAGAKI, T.; MIYAKAWA, M.: "Endometriosis of bladder after menopause". J. Urol., 145: 361, 1991.

61. SANCHEZ MERINO, J.M.; PARRA MUNTANER, L.; GUILLAN MAQUIEIRA, C. y cols.: "Bladder endometriosis". Arch. Esp. Urol., 52: 933, 1999. 
*62. FEDELE, L.; BIANCHI, S.; ZANCONATO, G. y cols.: "Use of a levonorgestrel-releasing intrauterine device in the treatment of rectovaginal endometriosis". Fertil Steril, 75: 485, 2001.

63. PICATOSTE, P.J.: "Endometriose vesicale". Acta Urol. Bel., 25: 318, 1957.

64. VICENTE, J.; ALGABA, F.: "Endometriosis vesical". Vicente J, Algaba F, editores. Semiología diagnostica endovesical, P 120-9. Pulso ediciones S.A. Barcelona, 1998.

65. PARRAS, M.; CASCON, E.; ROBLADO, R. y cols.: "Endometriosis vesical: utilidad de los métodos de imagen". Radiología, 41: 451, 1999.

66. ROMERO RONCEL, G.; BLASCO HERNANDEZ, P.; MARTINEZ RUIZ, R.M. y cols.: "Endometriosis urinaria: Revisión de nuestra casuística". Comunicación LXX Congreso Nacional de Urología, 2005.

67. LOPEZ MARTI, E.; PRATS LOPEZ, J.; PRERA VILASECA, A. y cols.: "Bladder endometriosis simulating a bladder tumor". Arch. Esp. Urol., 45: 158, 1992.

68. ROMERO PEREZ, P.; LOBATO ENCINAS, J.J.; PEREZ LLORCA, L.A. y cols.: "Endometriosis of the bladder muscular layer as a cause of acute abdomen". Arch. Esp. Urol., 48: 395, 1995.

69. PEREYRA, J.G.; ATECA, R.; GUTIERREZ, J.M. y cols.: "Endometriosis vesical". Video Arch. Esp. Urol., 12: 3, 2000.

70. CALDERAY, M.; GONZALEZ, J.M.; SANCHEZ, C. y cols.: "Endometriosis extragenital y extrapelviana". Prog. Obstet. Ginecol., 46: 458, 2003. 